\title{
24
}

\section{Towards a Theory of Sequential Hybrid Programs}

\author{
P.K. Pandya, H.-P. Wang and Q.-W. Xu \\ International Institute for Software Technology \\ United Nations University \\ P.O. Box 3058, Macau \\ E-mail: qxu@iist.unu.edu
}

\begin{abstract}
A theory of Sequential Hybrid Programs (SHP) is studied. SHP is a programming notation for representing hybrid systems. It contains a phase statement and the normal sequential programming constructs such as assignments, conditionals and iterations. Time dependent dynamical activities of the system are specified by phase statements. Intermixing of these two features leads to programs with a rich diversity of behaviours including super dense computations, infinite executions, finitely divergent executions and instantaneously divergent executions. Duration calculus is extended with super dense states, fixed point operators and infinite intervals to give a logic $\mu$ SDCI. A compositional semantics of SHP programs is defined using the logic $\mu$ SDCI. Several high level proof rules are derived for establishing specific kinds of properties of SHP programs such as total correctness and invariance. These high level proof rules provide a modular and syntax directed method for establishing the properties of SHP programs with the program structure guiding the proof of correctness.
\end{abstract}

\section{Keywords}

Duration Calculus, hybrid system, recursion, compositional semantics, modular verification

\section{INTRODUCTION}

Increased application of computer technology has led to novel computing systems which call for investigation of new modelling and verification techniques. Among these are the hybrid systems which consist of interacting discrete and continuous components. Typical examples of hybrid systems are embedded computer systems in which digital programs are used to control the physical devices. The physical states evolve over time in accordance with some dynamical laws, whereas discrete actions, such as computation steps or switches 
between phases of dynamical activities, are usually considered instantaneous. Such an abstraction provides an essential simplification in reasoning about hybrid systems (Manna \& Pnueli 1993).

This paper presents a theory for reasoning about hybrid systems using the framework of Duration Calculus (DC). A programming notation for hybrid systems, called Sequential Hybrid Programs (SHP), is proposed. It contains the usual constructs of sequential programming such as assignments, conditionals and iterations. These are called control actions. In addition, there are phase statements which model the time dependent dynamics. The control actions are assumed to take no time. Phase statements evolve according to some specified dynamical laws, and they may take time. Control actions govern the initiation and termination of phase statements. We use the Extended Duration Calculus (EDC) formulae (Zhou, Ravn \& Hansen 1993) to specify phase statements. Thus, phase statements may be used to specify the dynamics of a single continuous phase or the behaviours of a complex sub-program. Control actions contain the usual features of sequential programs, and these can be used to realise complex controllers incorporating numerical algorithms.

SHP programs can exhibit a rich variety of behaviours including super dense computations and infinite executions. Super dense computations arise when a number of instantaneous actions occur at the same time. Iteration can give rise to infinite executions. For example, depending on whether the body $P$ takes time, the iteration statement while $b$ do $P$ od can

(1) terminate in finite time after finitely many iterations,

(2) iterate a finite number of times and the last iteration does not terminate,

(3) iterate infinitely and the total execution time is not bounded,

(4) iterate infinitely and each iteration does not take time, or

(5) iterate infinitely and although infinitely many iterations takes nonzero time, the total execution time is bounded.

The fourth case, called instantaneous divergence, happens when the control program enters an infinite loop, or the system is switched repeatedly among several phases without spending any time in any of them. The last behaviour is the so called zeno behaviour, or finite divergence. For a more concrete impression of SHP programs, we refer to the toy example in Section 4.

In this paper, we give an adequate semantics which captures all the five behaviours listed above. It should be noted that although in general divergent behaviours are not desirable, to prove that they indeed do not occur, they must be modelled in the first place. For giving the semantics, we propose a version of DC with several additional features. The logic $\mu$ SDCI has super dense states, infinite intervals and recursion. By super dense states, we mean the system is defined as a function from time to a pair of states where the first state represents the conditions at the beginning of control actions, and the second state represents the conditions at the end of all the control actions oc- 
curring at that time point. The intermediate states are not recorded, because in a sequential setting, the internal details during the execution of control actions are unimportant. (There is some similarity with relational semantics for sequential programs here). Fixed point operators are used to model recursive behaviours and infinite intervals capture infinite executions.

In principle, the proof rules of $\mu$ SDCI can be used to derive properties of an SHP program as logical consequences of its semantics. However, this is usually not an effective method of reasoning, as the semantics can be complex. Instead, we propose a more high level modular approach. The low level logical semantics is used to derive several high level proof rules for establishing properties of interest. These include partial and total correctness, invariance and some temporal properties. Familiar logics such as Hoare Logic can be embedded within the proof method, and the normal patterns of syntax directed reasoning can be carried out using these. This is especially useful for reasoning about complex control actions, for they are modelled as sequential programs.

The rest of the paper is organised as follows. Section 2 defines the logic $\mu$ SDCI after briefly reviewing the classical DC. The SHP programs and their semantics are given in Section 3. The high level proof rules for modular verification are derived in Section 4 and applied to a toy example. The paper ends with a summary and discussion.

\section{LOGICS}

Duration Calculi are a family of real time logics, based on the Interval Temporal Logic (ITL) (Moszkowski 1985). Formulae are constructed from the following sets of symbols: A set of global variables $\operatorname{GVar}=\{x, y, \ldots\}$ and the meaning of a global variable is independent of time; A set of state variables $S \operatorname{Var}=\{S, U, \ldots\} ;$ A set of global function symbols $F S y m b=\left\{f^{n}, g^{m}, \ldots\right\}$, equipped with arities $n, m \geq 0$, and the meaning of a global function symbol $f^{n}, n>0$, is an $n$-ary function $\underline{f}^{n} \in R^{n} \rightarrow R$; A set of global relation symbols $R S y m b=\left\{G^{n}, H^{m}\right\}$, equipped with arities $n, m \geq 0$, and the meaning of a global relation symbol $G^{n}, n>0$, is an $n$-ary relation $\underline{G}^{n} \subseteq R^{n}$; A set of temporal propositional letters $P$ Letter $=\{X, Y, \ldots\}$.

The meaning of global variables is given by a value assignment,

$$
\mathcal{V} \in G \text { Var } \rightarrow \text { Values }
$$

associating a value with each global variable. In different variants of Duration Calculi, the interpretation of state variables and propositional letters are different.

\section{The Classical DC}


In the classical DC (Zhou, Hoare \& Ravn 1991), intervals, interpretations of state variables and propositional letters are defined as follows

$$
\begin{aligned}
& \text { Intv } \stackrel{\text { def }}{=}\{[c, d] \in \text { Time } \times \text { Time } \mid c \leq d\} \\
& \mathcal{I} \in S \text { Var } \rightarrow \text { Time } \rightarrow\{0,1\}, \quad \mathcal{J} \in \text { PLetters } \rightarrow \text { Intv } \rightarrow\{0,1\} .
\end{aligned}
$$

where Time denotes the set of non-negative reals. A model is a tuple of four elements $(\mathcal{I}, \mathcal{J}, \mathcal{V},[c, d])$. The terms and formulae are defined as follows. A Boolean state expression $B$ is constructed from (Boolean) state variables with Boolean connectives and its duration in a model

$$
\left(\int B\right)(\mathcal{I}, \mathcal{J}, \mathcal{V},[c, d]) \stackrel{\text { def }}{=} \int_{c}^{d}(B)(\mathcal{I}, \mathcal{V})(t) d t
$$

where $(B)(\mathcal{I}, \mathcal{V})(t)$ denotes the value of $B$ at time $t$ under state interpretation $\mathcal{I}$ and valuation $\mathcal{V}$. Primitive formulae of $\mathrm{DC}$ are constructed from terms using comparison operators in arithmetics, such as $<$, = etc, and can be combined by Boolean connectives and modality operators. The length $\ell$ of an interval in a model is defined as $\ell \stackrel{\text { def }}{=} \int 1$ and it is easy to prove that $(\ell)(\mathcal{I}, \mathcal{J}, \mathcal{V},[c, d])=d-c$. Boolean state expression $B$ holds almost everywhere (i.e., except possibly a finite number of points) over an interval, denoted as $\lceil B\rceil$, is defined as, $\lceil B\rceil \stackrel{\text { def }}{=} \int B=\ell \wedge \ell>0$. A point interval is characterised by $\ell=0$, shortened as $П$. The modality 'chop' of ITL is defined as follows: for any formulae $\alpha$ and $\beta$,

$$
\begin{aligned}
& (\mathcal{I}, \mathcal{J}, \mathcal{V},[c, d]) \vDash \alpha ; \beta \text { iff there exists } m \text { such that } c \leq m \leq d \text { and } \\
& \qquad(\mathcal{I}, \mathcal{J}, \mathcal{V},[c, m]) \models \alpha \text { and }(\mathcal{I}, \mathcal{J}, \mathcal{V},[m, d]) \models \beta .
\end{aligned}
$$

A property of DC, which becomes particularly important when extending the calculus with fixed point operators, is the following substitution lemma:

Substitution Lemma 1 For DC formulae $\alpha(X)$ and $\phi$, where $X$ is a propositional letter, $(\mathcal{I}, \mathcal{J}, \mathcal{V},[c, d]) \models \alpha(\phi / X)$ iff $\left(\mathcal{I}, \mathcal{J}^{\prime}, \mathcal{V},[c, d]\right) \models \alpha(X)$, where for any interval $[g, h], \mathcal{J}^{\prime}(X)([g, h])=1$ iff $(\mathcal{I}, \mathcal{J}, \mathcal{V},[g, h]) \models \phi$, and $\mathcal{J}^{\prime}(Y)([g, h])$ $=\mathcal{J}(Y)([g, h])$ for any $Y \neq X$. Note that substitution $\alpha(\phi / X)$ is not simple replacement of $X$ by $\phi$, because $\phi$ may contain free variables which become bound in $\alpha$. In such cases, the bound variables in $\alpha$ must be appropriately renamed. The precise definition of substitution can be found in standard text books, such as (Apt \& Olderog 1997).

\section{The SDC}

Hybrid systems contain physical variables which typically are of the type real. To describe and reason about hybrid systems, DC has been extended to 
EDC (Zhou, Ravn \& Hansen 1993), where state variables can be functions over arbitrary domains. To model state transitions, several of which may occur at one time point, DC is extended to SDC, where an interpretation of state variables is

$$
\mathcal{I} \in S \text { Var } \rightarrow \text { Time } \rightarrow \text { (Values } \times \text { Values }) .
$$

Let $S_{\mathcal{I}}$ denote the set of all state interpretations. Let $\mathcal{I}_{-1}$ and $\mathcal{I}_{-2}$ respectively be $\mathcal{I}$ projected to the first and the second values. At any point $t$ where $\mathcal{I}_{-1}$ or $\mathcal{I}_{-2}$ is continuous, we require that $\mathcal{I}_{-1}(S)(t)=\mathcal{I}_{-2}(S)(t)$. In the following diagram, the upper and lower curves represent respectively $\mathcal{I}_{-2}(S)(t)$ and $\mathcal{I}_{-1}(S)(t)$ as functions of time (but for visual convenience, we draw them slightly apart at continuous points).

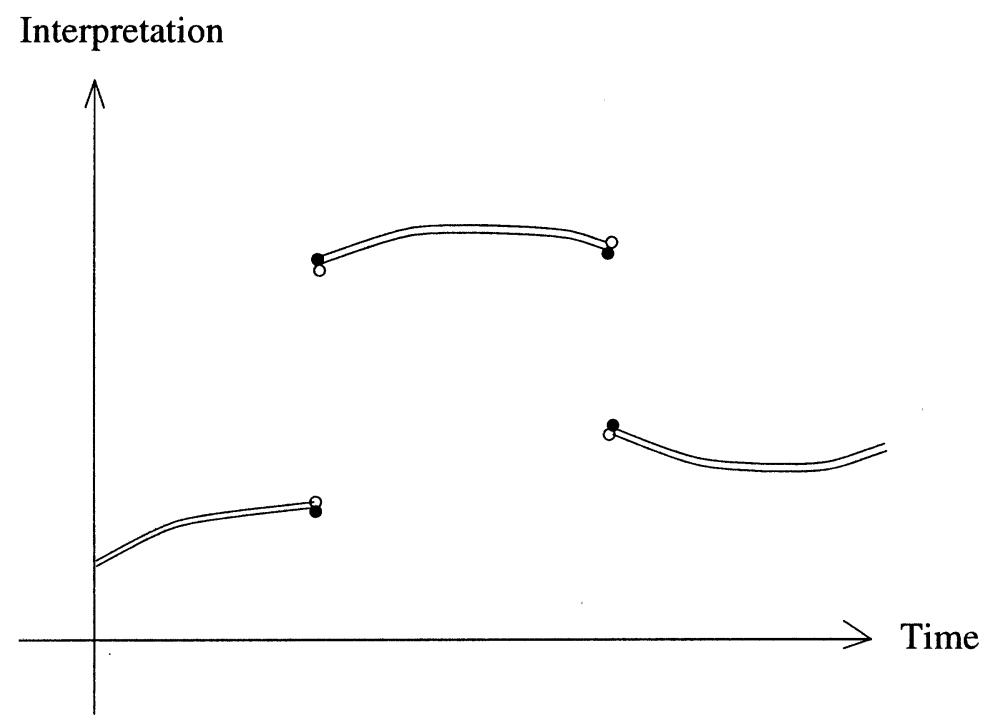

Fig. 1: Timing diagram of a state interpretation

Boolean state expressions, also called state predicates, can be constructed from state variables and global variables with usual comparison operators over the value domain and Boolean connectives. An example of a Boolean state expression could be (speed $>$ high) $\wedge$ brake_on, where speed and brake_on are state variables and high is a global variable. Two unary modalities are introduced to describe the pre- and post-conditions of discrete actions. For any model $(\mathcal{I}, \mathcal{J}, \mathcal{V},[c, d])$ and a Boolean state expression $B$, let

$$
\begin{array}{llll}
(\mathcal{I}, \mathcal{J}, \mathcal{V},[c, d]) \models \nwarrow B & \text { iff } & c=d \text { and } & (B)\left(\mathcal{I}_{-1}, \mathcal{V}\right)(c)=1 \\
(\mathcal{I}, \mathcal{J}, \mathcal{V},[c, d]) \models \nearrow B & \text { iff } & c=d \text { and } & (B)\left(\mathcal{I}_{-2}, \mathcal{V}\right)(c)=1
\end{array}
$$


where $(B)\left(\mathcal{I}_{-i}, \mathcal{V}\right)(c)$ denotes the value of $B$ at time $c$ under the interpretation $\mathcal{I}_{-i}$ and valuation $\mathcal{V}$. A state transition can be specified by a pair of pre- and post-conditions, e.g., $\nwarrow(x=0) \wedge \nearrow(x>8)$. The chop modality is defined as follows: for any SDC formulae $\alpha$ and $\beta$

$$
\begin{aligned}
(\mathcal{I}, \mathcal{J}, \mathcal{V},[c, d]) & \models \alpha ; \beta \text { iff there exist } m, \mathcal{I}^{\prime}, \mathcal{I}^{\prime \prime} \text { such that } c \leq m \leq d \text { and } \\
& \left(\mathcal{I}^{\prime},[c, m]\right) \otimes \mathcal{I}^{\mathcal{I}}\left(\mathcal{I}^{\prime \prime},[m, d]\right) \text { and } \\
& \left(\mathcal{I}^{\prime}, \mathcal{J}, \mathcal{V},[c, m]\right) \models \alpha \text { and }\left(\mathcal{I}^{\prime \prime}, \mathcal{J}, \mathcal{V},[m, d]\right) \vDash \beta
\end{aligned}
$$

where $\left(\mathcal{I}^{\prime},[c, m]\right) \otimes^{\mathcal{I}}\left(\mathcal{I}^{\prime \prime},[m, d]\right)$ iff for any state variable $S$

$$
\begin{aligned}
& \mathcal{I}^{\prime}(S)(t)=\mathcal{I}(S)(t) \text { if } c \leq t<m, \quad \mathcal{I}^{\prime}(S)(m)=\mathcal{I}_{-1}(S)(m), \\
& \mathcal{I}^{\prime \prime}(S)(t)=\mathcal{I}(S)(t) \text { if } m<t \leq d, \quad \mathcal{I}_{-2}^{\prime \prime}(S)(m)=\mathcal{I}_{-2}(S)(m), \\
& \text { and } \mathcal{I}_{-2}^{\prime}(S)(m)=\mathcal{I}_{-1}^{\prime \prime}(S)(m) .
\end{aligned}
$$

This chop modality satisfies the following properties

$$
\begin{aligned}
& \left(\nwarrow B_{1} \wedge \nearrow B_{2}\right) ;\left(\nwarrow B_{3} \wedge \nearrow B_{4}\right) \Leftrightarrow \nwarrow B_{1} \wedge \nearrow B_{4} \text { if } B_{2} \wedge B_{3} \text { is satisfiable, } \\
& \left(\nwarrow B_{1} \wedge \nearrow B_{2}\right) ;\left(\nwarrow B_{3} \wedge \nearrow B_{4}\right) \Leftrightarrow \text { false if } B_{2} \wedge B_{3} \text { is not satisfiable. }
\end{aligned}
$$

In Duration Calculi, state functions are usually assumed to satisfy the finite variability condition, which says that they are continuous almost everywhere, i.e., everywhere except for a finite number of points in any finite interval. Under this assumption, $\int_{c}^{d}(B)\left(\mathcal{I}_{-1}, \mathcal{V}\right)(t) d t=\int_{c}^{d}(B)\left(\mathcal{I}_{-2}, \mathcal{V}\right)(t) d t$, for a Boolean expression $B$, and we can define the duration of $B$ as

$$
\left(\int B\right)(\mathcal{I}, \mathcal{J}, \mathcal{V},[c, d]) \stackrel{\text { def }}{=} \int_{c}^{d}(B)\left(\mathcal{I}_{-1}, \mathcal{V}\right)(t) d t .
$$

For any term $z$ which only involves global variables and piece-wise continuous state function variables, $\lim _{t \rightarrow c^{-}}(z)\left(\mathcal{I}_{-1}, \mathcal{V}\right)(t)=\lim _{t \rightarrow c^{-}}(z)\left(\mathcal{I}_{-2}, \mathcal{V}\right)(t)$ and $\lim _{t \rightarrow c^{+}}(z)\left(\mathcal{I}_{-1}, \mathcal{V}\right)(t)=\lim _{t \rightarrow c^{+}}(z)\left(\mathcal{I}_{-2}, \mathcal{V}\right)(t)$. We define the limit values of such a term at the beginning and the end of a non-empty interval as

$$
\begin{aligned}
& (\mathbf{b} . z)(\mathcal{I}, \mathcal{J}, \mathcal{V},[c, d]) \stackrel{\text { def }}{=} \lim _{t \rightarrow c^{+}}(z)\left(\mathcal{I}_{-1}, \mathcal{V}\right)(t), \\
& (\mathbf{e} . z)(\mathcal{I}, \mathcal{J}, \mathcal{V},[c, d]) \stackrel{\text { def }}{=} \lim _{t \rightarrow d^{-}}(z)\left(\mathcal{I}_{-1}, \mathcal{V}\right)(t)
\end{aligned}
$$

The definition of $\lceil B\rceil$ remains the same, i.e., $\lceil B\rceil \stackrel{\text { def }}{=} \int B=\ell \wedge \ell>0$.

In EDC, the following MT rule allows mathematical theories to be imported into the calculus:

Let $R(\lceil H(z)\rceil$, b. $z, \mathbf{e} . z, l)$ be a EDC formula without chop.

If in mathematics $(\forall c \leq d) R\left(\forall c \leq t \leq d . H(z(t)), z\left(c^{+}\right), z\left(d^{-}\right), d-c\right)$ then \lceil\rceil $R(\lceil H(z)\rceil, \mathbf{b} . z, \mathbf{e} . z, \ell)$ 
where $z\left(c^{+}\right)$and $z\left(d^{-}\right)$denote limits of $z$ from the right of $c$ and left of $d$ respectively.

Substitution Lemma 1, as stated in the last section, is not valid for SDC. Below is a counterexample: let $S$ be a state variable and $\mathcal{I}_{-2}(S)(t)=0$ for any $t$, we have $(\mathcal{I}, \mathcal{J}, \mathcal{V},[c, d]) \vDash \nearrow S$; true, but $\left(\mathcal{I}, \mathcal{J}^{\prime}, \mathcal{V},[c, d]\right) \not X X$; true, because for any interval $[g, h], \mathcal{J}^{\prime}(X)([g, h])=0$ as $(\mathcal{I}, \mathcal{J}, \mathcal{V},[g, h]) \forall \nearrow S$. The complication in SDC is that in the definition of chop, not only the interval but also the state interpretation $\mathcal{I}$ may be changed. Therefore, the interpretation of propositional letters should be defined as

$$
\mathcal{J} \in \text { PLetters } \rightarrow\left(S_{\mathcal{I}} \times \text { Intv }\right) \rightarrow\{0,1\}
$$

Under the new definition, we have

Substitution Lemma 2 For SDC formulae $\alpha(X)$ and $\phi,(\mathcal{I}, \mathcal{J}, \mathcal{V},[c, d]) \vDash$ $\alpha(\phi)$ iff $\left(\mathcal{I}, \mathcal{J}^{\prime}, \mathcal{V},[c, d]\right) \models \alpha(X)$, where for any interpretation of state variables $\mathcal{I}^{\prime}$ and interval $[g, h], \mathcal{J}^{\prime}(X)\left(\mathcal{I}^{\prime},[g, h]\right)=1$ iff $\left(\mathcal{I}^{\prime}, \mathcal{J}, \mathcal{V},[g, h]\right) \models \phi$ and $\mathcal{J}^{\prime}(Y)\left(\mathcal{I}^{\prime},[g, h]\right)=\mathcal{J}(Y)\left(\mathcal{I}^{\prime},[g, h]\right)$ for any $Y \neq X$.

\section{$\mu$ SDCI}

The standard way to describe the semantics of a recursive program is to define it as a fixed point (Scott \& Strachey 1971) of an appropriate functional. Fixed points operators have also been introduced in propositional and modal logics, see e.g., (Kozen \& Parikh 1981, Stirling 1995), and in Duration Calculus (Pandya \& Ramakrishna 1995). In this section, we propose an extension of SDC, called $\mu$ SDCI, which includes fixed points operators and infinite intervals. The syntax of $\mu$ SDCI contains two additional constructs $(\mu X) \alpha$ and $(\nu X) \alpha$, where the occurrence of $X$ in $\alpha$ is positive (i.e., $X$ is preceded by even number of negation symbols).

To give the semantics of fixed point operators by Knaster-Tarski theorem, we consider the complete lattice $\left(2^{\left(S_{\mathcal{I}} \times \operatorname{Intv}\right)}, \subseteq\right)$, where

$$
\begin{aligned}
& \text { Time }^{+} \stackrel{\text { def }}{=} \text { Time } \cup\{\infty\}, \\
& \text { Intv } \stackrel{\text { def }}{=}\left\{[c, d] \in \text { Time } \times \text { Time }^{+} \mid c \leq d\right\} .
\end{aligned}
$$

For notational convenience, we regard an interpretation of propositional letters $\mathcal{J}$ as a function in PLetters $\rightarrow 2^{\left(S_{\mathcal{I} \times I n t v)}\right.}$. For a given interpretation of propositional letters $\mathcal{J}$, a valuation of global variables $\mathcal{V}$, we define a function $\mathcal{E}_{\mathcal{V}}^{\mathcal{J}}$ from the set of $\mu$ SDCI formulae to $2^{\left(S_{\mathcal{I}} \times \operatorname{Intv}\right)}$ by induction:

$$
\begin{aligned}
& \mathcal{E}_{\mathcal{V}}^{\mathcal{J}}\left(G^{n}\left(z_{1}, \ldots, z_{n}\right)\right) \stackrel{\text { def }}{=} \\
& \quad\left\{(\mathcal{I},[c, d]) \mid\left(\left(z_{1}\right)(\mathcal{I}, \mathcal{J}, \mathcal{V},[c, d]), \ldots,\left(z_{n}\right)(\mathcal{I}, \mathcal{J}, \mathcal{V},[c, d])\right) \in \underline{G}^{n}\right\} \\
& \quad \text { where }\left(z_{i}\right)(\mathcal{I}, \mathcal{J}, \mathcal{V},[c, d]) \text { denotes the value of term } z_{i} \text { in the model }
\end{aligned}
$$




$$
\begin{aligned}
& \mathcal{E}_{\mathcal{V}}^{\mathcal{J}}(X) \stackrel{\text { def }}{=} \mathcal{J}(X) \\
& \mathcal{E}_{\mathcal{V}}^{\mathcal{J}}(\nwarrow B) \stackrel{\text { def }}{=}\left\{(\mathcal{I},[c, d]) \mid c=d \neq \infty \text { and }(B)\left(\mathcal{I}_{-1}, \mathcal{V}\right)(c)=1\right\} \text {, } \\
& \mathcal{E}_{\mathcal{V}}^{\mathcal{J}}(\nearrow B) \stackrel{\text { def }}{=}\left\{(\mathcal{I},[c, d]) \mid c=d \neq \infty \text { and }(B)\left(\mathcal{I}_{-2}, \mathcal{V}\right)(c)=1\right\} \text {, } \\
& \mathcal{E}_{\mathcal{V}}^{\mathcal{J}}(\neg \alpha) \stackrel{\text { def }}{=} 2^{\left(S_{\mathcal{I}} \times \text { Intv }\right)}-\mathcal{E}_{\mathcal{V}}^{\mathcal{J}}(\alpha) \\
& \mathcal{E}_{\mathcal{V}}^{\mathcal{J}}\left(\alpha_{1} \vee \alpha_{2}\right) \stackrel{\text { def }}{=} \mathcal{E}_{\mathcal{V}}^{\mathcal{J}}\left(\alpha_{1}\right) \cup \mathcal{E}_{\mathcal{V}}^{\mathcal{J}}\left(\alpha_{2}\right), \\
& \mathcal{E}_{\mathcal{V}}^{\mathcal{J}}\left(\alpha_{1} ; \alpha_{2}\right) \stackrel{\text { def }}{=}\left(\mathcal{E}_{\mathcal{V}}^{\mathcal{J}}\left(\alpha_{1}\right) \cap\{(\mathcal{I},[c, d]) \mid c \in \text { Time, } d=\infty\}\right) \\
& \cup\left(\mathcal{E}_{\mathcal{V}}^{\mathcal{J}}\left(\alpha_{1}\right) \otimes \mathcal{E}_{\mathcal{V}}^{\mathcal{J}}\left(\alpha_{2}\right)\right) \\
& \mathcal{E}_{\mathcal{V}}^{\mathcal{J}}((\nu X) \alpha)=\bigcup\left\{\chi \mid \mathcal{E}_{\mathcal{V}}^{\mathcal{J}(X \mapsto \chi)}(\alpha) \supseteq \chi\right\} \text {. }
\end{aligned}
$$

The set theoretical semantics is related to satisfaction and validity in obvious ways, namely, for any $\alpha$,

$$
\begin{aligned}
& (\mathcal{I}, \mathcal{J}, \mathcal{V},[c, d]) \models \alpha \text { iff }(\mathcal{I},[c, d]) \in \mathcal{E}_{\mathcal{V}}^{\mathcal{J}}(\alpha) \\
& \vDash \alpha \text { iff } \mathcal{E}_{\mathcal{V}}^{\mathcal{J}}(\alpha)=2^{\left(S_{\mathcal{I}} \times \operatorname{Intv}\right)} \text { for any } \mathcal{J} \text { and } \mathcal{V}
\end{aligned}
$$

In this setting, the substitution lemma is expressed as

$$
\mathcal{E}_{\mathcal{V}}^{\mathcal{J}}(\alpha(\phi))=\mathcal{E}_{\mathcal{V}}^{\mathcal{J}\left(X \mapsto \mathcal{E}_{\mathcal{V}}^{\mathcal{J}}(\phi)\right)}(\alpha(X))
$$

Finite and infinite intervals are characterised by

$$
\text { inf } \stackrel{\text { def }}{=}(\forall x)(\ell \geq x), \quad \text { fin } \stackrel{\text { def }}{=} \neg \text { inf. }
$$

It is easy to prove the following theorems

$$
\begin{array}{ll}
\models((\alpha \wedge \text { inf }) ; \beta) \Leftrightarrow(\alpha \wedge \text { inf }), & \models \text { inf } \Leftrightarrow \text { (true; false), } \\
\models \text { inf; } \alpha \Rightarrow \text { inf }, & \models \alpha ; \text { inf } \Rightarrow \text { inf. }
\end{array}
$$

For any interpretation of propositional letters $\mathcal{J}$, global valuation $\mathcal{V}, \mu$ SDCI formula $\alpha$ and propositional letter $X$, let $\left(F_{\mathcal{V}}^{\mathcal{J}}\right)_{X}^{\alpha}$ be the function in $2^{\left(S_{\mathcal{I}} \times \operatorname{Intv}\right)} \longrightarrow$ $2^{\left(S_{\mathcal{I}} \times \operatorname{Intv}\right)}$ such that:

$$
\left(F_{\mathcal{V}}^{\mathcal{J}}\right)_{X}^{\alpha}: \quad \chi \longmapsto \mathcal{E}_{\mathcal{V}}^{\mathcal{J}(X \mapsto \chi)}(\alpha) \quad \text { for any } \chi \in 2^{\left(S_{\mathcal{I} \times \operatorname{Intv})}\right.}
$$

It is easy to prove that if $X$ is positive in $\alpha$ then $\left(F_{\mathcal{V}}^{\mathcal{J}}\right)_{X}^{\alpha}$ is monotonic with 
respect to the set inclusion order. Therefore by Knaster-Tarski fixed point theorem, we have

Theorem 1 For any interpretation of propositional letters $\mathcal{J}$, valuation $\mathcal{V}$, $\mu S D C I$ formula $\alpha$ and propositional letter $X, \mathcal{E}_{\mathcal{V}}^{\mathcal{J}}((\mu X) \alpha)$ and $\mathcal{E}_{\mathcal{V}}^{\mathcal{J}}((\nu X) \alpha)^{*}$ are respectively the least and greatest fixed points of $\left(F_{\mathcal{V}}^{\mathcal{J}}\right)_{X}^{\alpha}$ in $\left(2^{\left(S_{\mathcal{I}} \times I n t v\right)}, \subseteq\right)$.

It follows from this theorem and Substitution Lemma 2 that

Theorem 2 For any $\mu S D C I$ formula $(\mu X) \alpha$, $\vDash \alpha((\mu X) \alpha) \Leftrightarrow(\mu X) \alpha$, and $\vDash \alpha((\nu X) \alpha) \Leftrightarrow(\nu X) \alpha$.

Theorem 3 Let $\alpha$ be a $\mu S D C I$ formula and $X$ a propositional letter such that the occurrence of $X$ in $\alpha$ is positive.

(1) If $\models \alpha(\phi / X) \Rightarrow \phi$ then $\vDash(\mu X) \alpha \Rightarrow \phi$;

(2) If $\models \phi \Rightarrow \alpha(\phi / X)$ then $\vDash \phi \Rightarrow(\nu X) \alpha$.

Theorem 4 Let $X$ be a propositional variable, $\alpha_{1}$ and $\alpha_{2}$ be two formulae in which $X$ is positive. If $\models \alpha_{1} \Rightarrow \alpha_{2}$, then $\models\left((\mu X) \alpha_{1}\right) \Rightarrow\left((\mu X) \alpha_{2}\right)$ and $\vDash\left((\nu X) \alpha_{1}\right) \Rightarrow\left((\nu X) \alpha_{2}\right)$.

Theorem 5 Let $\alpha, \phi$ and $\psi$ be $\mu$ SDCI formulae, and $X$ a propositional letter positive in $\alpha$. If $\models \phi \Rightarrow \psi$, then $\models \alpha(\phi / X) \Rightarrow \alpha(\psi / X)$.

The last four theorems provide some simple syntactic rules for reasoning about $\mu \mathrm{SDCI}$ formulae. For example, they can be used to prove that the following formulae are valid.

\section{Example 1}

$$
\begin{array}{ll}
(\mu X)(\text { true } ; X) \Leftrightarrow \text { inf, } & (\nu X)(\text { true } X) \Leftrightarrow \text { true } \\
(\mu X)(\text { inf } ; X) \Leftrightarrow \text { inf, } & (\nu X) \text { (inf; } X) \Leftrightarrow \text { inf } \\
(\mu X)(\text { fin } ; X) \Leftrightarrow \text { false, } & (\nu X)(\text { fin } ; X) \Leftrightarrow \text { true }, \\
(\mu X)(X ; \alpha) \Leftrightarrow \text { false, } & (\nu X)(\alpha ;(X \wedge \text { inf })) \Rightarrow \text { inf. }
\end{array}
$$

\section{SEQUENTIAL HYBRID PROGRAMS}

We propose a language for describing hybrid systems based on the earlier work in (Xu 1997). The language supports constructive design by allowing specification statements to be mixed with program constructs, in the tradition of the well established theories of program development such as refinement 
calculus (Back 1980, Morgan 1990). In addition to usual program constructs, the language includes a specification statement for describing (instantaneous) state transitions and a specification statement using an EDC formula for describing a possibly hybrid component with time dependent dynamics.

The abstract syntax and informal interpretation of the basic commands are as follows, where for simplicity, we assume $\bar{x}$ contains all the system variables.

$$
P::=\left\langle R\left(\bar{x}, \bar{x}^{\prime}\right)\right\rangle|\ll C \gg| P_{1} ; P_{2}\left|P_{1}[] P_{2}\right| \text { while } B \text { do } P \text { od. }
$$

$\left\langle R\left(\bar{x}, \bar{x}^{\prime}\right)\right\rangle$ is the nondeterministic assignment; its execution is instantaneous and the state transition satisfies the binary predicate $R$; Sequential composition is represented by $P_{1} ; P_{2}$ and its meaning is that if $P_{1}$ terminates then $P_{2}$ is executed immediately afterwards; [] is the nondeterministic choice; Statement $\ll C \gg$ describes a component by an EDC formula $C$. Continuous evolution can be specified by an invariant expressing the dynamical laws. The effect of the statement is like skip if no time is elapsed during its execution; Iteration statement is executed repeatedly until the boolean guard becomes false. Other commands can be defined from the basic ones, e.g.,

$$
\begin{aligned}
& \bar{x}:=\exp (\bar{x}) \stackrel{\text { def }}{=}\left\langle\bar{x}^{\prime}=\exp (\bar{x})\right\rangle, \\
& \text { skip } \stackrel{\text { def }}{=} x:=x \text {, } \\
& \text { if } B_{1}(\bar{x}) \rightarrow P_{1} \text { [] } \cdots \text { [] } B_{n}(\bar{x}) \rightarrow P_{n} \text { fi } \\
& \stackrel{\text { def }}{=}\left(\left\langle B_{1}(\bar{x}) \wedge \bar{x}^{\prime}=\bar{x}\right\rangle ; P_{1}\right)[] \cdots[]\left(\left\langle B_{n}(\bar{x}) \wedge \bar{x}^{\prime}=\bar{x}\right\rangle ; P_{n}\right) \text {, } \\
& \text { delay } t \stackrel{\text { def }}{=} \ll l=t \gg \text {. }
\end{aligned}
$$

We shall abbreviate $\left\langle B(\bar{x}) \wedge \bar{x}^{\prime}=\bar{x}\right\rangle$ as $\langle B(\bar{x})\rangle$. The semantics is defined as follows

$$
\begin{aligned}
& \llbracket\left\langle R\left(\bar{x}, \bar{x}^{\prime}\right)\right\rangle \rrbracket \stackrel{\text { def }}{=}(\exists \bar{u} \bar{v})(R(\bar{u}, \bar{v}) \wedge \nwarrow(\bar{x}=\bar{u}) \wedge \nearrow(\bar{x}=\bar{v})), \\
& \llbracket \ll C \gg \rrbracket \stackrel{\text { def }}{=}(\exists \bar{u} \bar{v})(\nwarrow(\bar{x}=\bar{u}) \\
& ;(\mathbf{b} . \bar{x}=\bar{u} \wedge C \wedge(\ell>0) \wedge \mathbf{e} \cdot \bar{x}=\bar{v}) \\
& ; \nearrow(\bar{x}=\bar{v})) \\
& \vee(\exists \bar{v})(\nwarrow(\bar{x}=\bar{v}) \wedge \nearrow(\bar{x}=\bar{v}) \wedge C \wedge(\ell=0)), \\
& \llbracket P_{1} ; P_{2} \rrbracket \stackrel{\text { def }}{=} \llbracket P_{1} \rrbracket ; \llbracket\left[P_{2} \rrbracket\right. \text {, } \\
& \| P_{1}[] P_{2} \rrbracket \stackrel{\text { def }}{=} \llbracket P_{1} \rrbracket \vee \llbracket P_{2} \rrbracket \text {, } \\
& \text { \|while } B \text { do } P \text { od } \rrbracket \stackrel{\text { def }}{=}(\mu X)((\llbracket\langle B\rangle \rrbracket ; \llbracket P \rrbracket ; X) \vee \llbracket\langle\neg B\rangle]) \\
& \vee(\nu X)(((\llbracket\langle B\rangle] ;[P]) \wedge \text { fin }) ;(X \wedge \text { inf })) \text {. }
\end{aligned}
$$

The semantics of the iteration statement needs some elaboration. The least fixed point $(\mu X)((\llbracket\langle B\rangle \rrbracket ; \| P \rrbracket ; X) \vee \llbracket\langle\neg B\rangle \rrbracket)$ characterises the executions with finitely many iterations of the loop, where the Boolean guard becomes false eventually or the last iteration takes infinite time. We take the view 
that if the body does not take time, then finitely many iterations of the loop also do not take time. The least fixed point formula evaluated to false if the number of iterations is infinite. The greatest fixed point $(\nu X)(((\llbracket\langle B\rangle] ;[P]) \wedge$ fin); $(X \wedge$ inf $))$, on the other hand, describes executions with infinitely many iterations. Normally, infinitely many iterations altogether take infinite time. In our model, we also record divergent behaviours which arise either because infinitely many iterations of instantaneous actions or because infinitely many iterations with total execution time bounded (e.g., if the $n$th iteration takes time $\frac{1}{2^{n}}$, then within 2 units there will be infinitely many iterations). We follow a CSP-like approach (Hoare 1985) to divergence and consider the behaviour after infinitely many iterations as nonterminating but totally arbitrary, and model it by the formula inf.

The above modelling of divergence can be justified as follows: We regard infinitely many iterations of instantaneous actions undesirable, because if this happens the plant will not receive any control signals. The other case of zeno behaviour also seems undesirable, and therefore we consider the behaviour after the limit time point of a zeno computation arbitrary. This view is technically convenient, and certainly safe. We have not yet come across serious applications with zeno computations either to test or to challenge it. It appears that to model zeno behaviour more accurately, the notion of limit of a convergent sequence of reals has to be incorporated into the logic (Hansen, Pandya \& Zhou 1995).

Example 2 We show $\| x:=x+1 ; x:=x+2 \rrbracket \Leftrightarrow \llbracket[x:=x+3 \rrbracket$.

This is because

$$
\begin{aligned}
& \mathbb{}[x:=x+1 ; x:=x+2 \rrbracket \\
& \Leftrightarrow \llbracket x:=x+1 \rrbracket ; \llbracket x:=x+2 \rrbracket \\
& \Leftrightarrow((\exists v)(\nwarrow(x=v) \wedge \nearrow(x=v+1))) ;((\exists v)(\nwarrow(x=v) \wedge \nearrow(x=v+2))) \\
& \Leftrightarrow\left(\exists v_{1}, v_{2}\right)\left(\left(\nwarrow\left(x=v_{1}\right) \wedge \nearrow\left(x=v_{1}+1\right)\right) ;\left(\nwarrow\left(x=v_{2}\right) \wedge \nearrow\left(x=v_{2}+2\right)\right)\right) \\
& \Leftrightarrow\left(\exists v_{1}, v_{2}\right)\left(\left(v_{1}+1=v_{2}\right) \wedge \nwarrow\left(x=v_{1}\right) \wedge \nearrow\left(x=v_{2}+2\right)\right) \\
& \Leftrightarrow(\exists v)(\nwarrow(x=v) \wedge \nearrow(x=v+3)) \\
& \Leftrightarrow \mathbb{x}:=x+3] .
\end{aligned}
$$

Example 3 We show [while true do skip od] $\Leftrightarrow$ inf.

We have

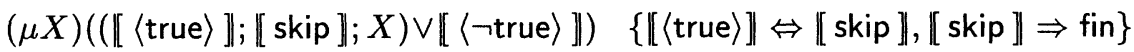

$$
\begin{aligned}
& \Rightarrow(\mu X)(\text { fin; } X) \\
& \Leftrightarrow \text { false } \\
& \text { \{Example } 1 \text { in previous section }
\end{aligned}
$$

and $(\nu X)(((\llbracket\langle$ true $\rangle] ; \llbracket$ skip $]) \wedge$ fin $) ;(X \wedge$ inf $)) \Leftrightarrow(\nu X)(\|$ skip $\rrbracket ;(X \wedge$ inf $))$. Because inf $\Rightarrow \llbracket$ skip $\rrbracket ;$ (inf $\wedge$ inf), it follows from Theorem 3 that inf $\Rightarrow$ $(\nu X)(\llbracket$ skip $\rrbracket ;(X \wedge$ inf $))$. Example 1 in the previous section shows that $(\nu X)$ ([skip $] ;(X \wedge$ inf $)) \Rightarrow$ inf. Therefore, $[$ while true do skip od $] \Leftrightarrow$ inf.

Unfolding Law The iteration statement can be unfolded as usual, formally, 
$\vDash \alpha(\| W \rrbracket) \Leftrightarrow \|[W]$, where $\alpha(X)=(\|\langle B\rangle \rrbracket ;\| P \rrbracket ; X) \vee \|\langle\neg B\rangle \rrbracket, \beta(X)=$ $((\|\langle B\rangle \rrbracket ;[P]\| \wedge$ fin $) ;(X \wedge$ inf $)$ and $\llbracket W \rrbracket=((\mu X) \alpha(X)) \vee((\nu X) \beta(X))$.

\section{MODULAR VERIFICATION}

To effectively reason about SHP programs, we use the familiar notations from Hoare logic. Let $p$ and $q$ be state predicates, and $\alpha$ a formula. Define

$$
\{p\} P\{q\} \stackrel{\text { def }}{=} \nearrow p ;\|P\| \Rightarrow \text { fin; } \nearrow q, \quad\{p\} P \alpha \stackrel{\text { def }}{=} \nearrow p ; \| P \rrbracket \Rightarrow \alpha
$$

\subsection{Total Correctness}

A discrete component is an SHP statement which does not use the phase statement $\ll C \gg$. Consequently, if the execution is not divergent, it takes no time. For discrete components, the Hoare triple $\{p\} \quad D \quad\{q\}$ of total correctness is equivalent to

$$
\nearrow p ;[D] \Rightarrow \nearrow q
$$

Rules of Hoare Logic for sequential discrete programs can be considered as abbreviations of $\mu$ SDCI theorems.

$$
\begin{aligned}
& \{p\}<R\left(\bar{x}, \bar{x}^{\prime}\right)>\{(\exists \bar{v}) p(\bar{v}) \wedge R(\bar{v}, \bar{x})\} \\
& \nearrow p ;(\exists \bar{u} \bar{v})(R(\bar{u}, \bar{v}) \wedge \nwarrow(\bar{x}=\bar{u}) \wedge \nearrow(\bar{x}=\bar{v})) \Rightarrow \nearrow((\exists \bar{v}) p(\bar{v}) \wedge R(\bar{v}, \bar{x})) \\
& \frac{\{p\} D_{1}\{r\} \quad\{r\} D_{2}\{q\}}{\{p\} D_{1} ; D_{2}\{q\}} \\
& \frac{\nearrow p ; \llbracket\left[D_{1} \rrbracket \Rightarrow \nearrow r \quad \nearrow r ;\left[D_{2} \rrbracket \Rightarrow \nearrow q\right.\right.}{\left.\nearrow p ; \| D_{1} ; D_{2}\right] \Rightarrow \nearrow q} \\
& \frac{\{p\} D_{1}\{q\} \quad\{p\} D_{2}\{q\}}{\{p\} D_{1}[] D_{2}\{q\}} \\
& \frac{\nearrow p ; \llbracket D_{1} \rrbracket \Rightarrow \nearrow q \quad \nearrow p ; \llbracket D_{2} \rrbracket \Rightarrow \nearrow q}{\nearrow p ; \llbracket D_{1}[] D_{2} \rrbracket \Rightarrow \nearrow q} \\
& r(i) \wedge i>0 \Rightarrow B \quad r(0) \Rightarrow \neg B \\
& \frac{\{r(i) \wedge i>0\} D\{(\exists j<i) r(j)\}}{\{\exists i . r(i)\} \text { while } B \text { do } D \text { od }\{r(0)\}} \\
& r(i) \wedge i>0 \Rightarrow B \quad r(0) \Rightarrow \neg B \\
& \frac{\nearrow(r(i) \wedge i>0) ;[D \rrbracket \Rightarrow \nearrow((\exists j<i) r(j))}{\nearrow(\exists i . r(i)) ; \llbracket \text { while } B \text { do } D \text { od } \rrbracket \Rightarrow \nearrow r(0)}
\end{aligned}
$$




$$
\begin{aligned}
& \frac{p \Rightarrow p_{1} \quad\left\{p_{1}\right\} D\left\{q_{1}\right\} \quad q_{1} \Rightarrow q}{\{p\} D\{q\}} \\
& \frac{p \Rightarrow p_{1} \quad \nearrow p_{1} ;[D] \Rightarrow \nearrow q_{1} \quad q_{1} \Rightarrow q}{\nearrow p ; \|[D] \Rightarrow \nearrow q}
\end{aligned}
$$

We only prove the soundness of iteration rule. Let $W \stackrel{\text { def }}{=}$ while $B$ do $D$ od.

$$
\begin{array}{ll}
\nearrow r(0) ; \llbracket W \rrbracket & \text { \{unfolding law } \\
\Rightarrow \nearrow r(0) ;((\llbracket\langle B\rangle \rrbracket ; \| D \rrbracket ; \llbracket W \rrbracket) \vee \llbracket\langle\neg B\rangle \rrbracket) & \\
\Leftrightarrow \nearrow r(0) . &
\end{array}
$$

For any $i>0$

$$
\begin{aligned}
& \nearrow r(i) ; \| W \rrbracket \\
& \Rightarrow \nearrow r(i) ;((\|\langle B\rangle] ; \|[D] ;[[W]) \vee \llbracket\langle\neg B\rangle]) \\
& \Leftrightarrow \nearrow r(i) ;([\langle B\rangle] ;[[D] ;[[W]) \\
& \Rightarrow(\exists j<i) \nearrow r(j) ; \| W] .
\end{aligned}
$$

\{unfolding law\} $\{r(i) \Rightarrow B\}$

It follows from induction that $\nearrow(\exists i . r(i)) ; \llbracket[W \rrbracket \Rightarrow \nearrow r(0) ;[[W \rrbracket$ and subsequently $\nearrow(\exists i . r(i)) ;[W] \Rightarrow \nearrow r(0)$.

\subsection{Temporal Properties}

We propose a number of rules which can be used to verify properties that are not restricted to pre-conditions and post-conditions. In the following, $P, P_{1}$ and $P_{2}$ may contain continuous components.

$$
\begin{aligned}
& \{\text { true }\}<R\left(\bar{x}, \bar{x}^{\prime}\right)>\prod
\end{aligned}
$$

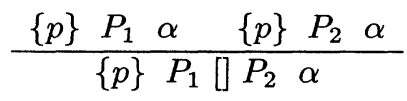

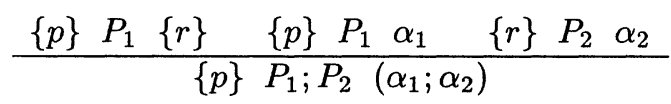

$$
\begin{aligned}
& \frac{\{p\} P_{1}(\alpha \wedge \text { inf })}{\{p\} P_{1} ; P_{2}(\alpha \wedge \text { inf })}
\end{aligned}
$$

One particularly interesting property is invariance, expressed as $\lceil S\rceil \vee\lceil\rceil$. The following rule says that an iteration statement satisfies an invariant if the 
body always takes at least some non-zero constant time $c$ (this ensures that there is no divergence or zeno) and satisfies the invariant.

$$
\frac{\{B \wedge r\} P((\lceil S\rceil \wedge l>c>0) ; \nearrow r)}{\{r\} \text { while } B \text { do } P \text { od }(\lceil S\rceil \vee\lceil\rceil)}
$$

The correctness of the invariant rule follows from the unfolding law.

$$
\begin{array}{ll}
\nearrow r ;[\| W \rrbracket & \text { \{unfolding law } \\
\Rightarrow \nearrow r ;((\|\langle B\rangle] ;\|P \rrbracket ;\| W \|) \vee \llbracket\langle\neg B\rangle]) & \\
\Rightarrow(\lceil S\rceil \wedge l>c>0) ; \nearrow r ; \| W \rrbracket \vee\lceil S\rceil \vee \sqcap . &
\end{array}
$$

By induction

$$
\begin{aligned}
& \nearrow r ;[W] \\
& \Rightarrow(\forall n)((\lceil S\rceil \wedge l>n \times c \wedge c>0) ; \nearrow r ;[W]) \vee\lceil S\rceil \vee \sqcap \\
& \Rightarrow\lceil S\rceil \vee\lceil.
\end{aligned}
$$

\subsection{A Simple Example}

We consider the following toy example, named CRUISE for convenience in exposition. In the system, the current speed of the car $V$ is continuous and is controlled by a computer by periodically calculating the acceleration $a$ which must be applied for the next time unit. The sampling of the current speed, is denoted by the statement $v:=V$. If $v<80$, the car can accelerate and the rate of acceleration is computed by a control algorithm $A$. Similarly, if $v \geq 70$, the car may decelerate at a rate determined by a control algorithm $D$. When accelerating, the behaviour of the car is defined by the phase statement $\ll \exists x . x=$ b. $a \wedge\lceil|\dot{V}-x| \leq 3 \wedge \dot{V} \geq 1\rceil \wedge \ell=1 \gg$. Due to hardware limitations, the actual acceleration $\dot{V}$ may not be exactly the same as specified. Condition $|\dot{V}-x| \leq 3 \wedge \dot{V} \geq 1$ states that $\dot{V}$ is within an error of 3 from the value of $x$ and that it is at least 1 . The design task is to construct components $A$ and $D$ such that the speed never exceeds 100 .

The requirement can be expressed by the following correctness formula

$$
\text { true\} CRUISE }(\lceil V<100\rceil) \vee\lceil\rceil)
$$

Due to compositionality of our method, we can verify the correctness of the system based on the specifications of $A$ and $D$. We record the verification by what is commonly called proof outline in program verification. In the outline, the formulae, marked by shaded boxes, represent conditions and conclusions of various verification rules used. For example, the formula in line (2) is obtained by applying the assignment axiom to the statement in line (1). The formula 
in turn serves as the pre-condition for the statement in line (3). Formula in line (6) is carried over from line (4), and is used as the pre-condition of the iteration body; line (19) denotes the property that the body has, and by the iteration rule, the iteration statement satisfies the property in line (21).

$$
\begin{aligned}
& V:=0 \\
& \{V=0\} \\
& \text { alarm := off; } \\
& \{V<100\} \\
& \text { while true do } \\
& \{V<100\} \\
& v:=V \text {; } \\
& \{V<100 \wedge v=V\} \\
& \text { if } v<80 \rightarrow\{V<80\} A ;\{V<80 \wedge a<15\} \\
& \ll \exists x . x=\text { b. } a \wedge\lceil|\dot{V}-x| \leq 3 \wedge \dot{V} \geq 1\rceil \wedge \ell=1 \gg \\
& (\lceil V<100] \wedge l>0.5) ; \nearrow(V<100) \\
& \text { [] } v \geq 70 \rightarrow\{V<100\} D ;\{V<100\} \\
& \ll \exists x . x=\text { b. } a \wedge\lceil|\dot{V}-x| \leq 3 \wedge \dot{V} \leq-1\rceil \wedge \ell=1 \gg \\
& ([V<100] \wedge l>0.5) ; \nearrow(V<100) \\
& \text { [] } v>100 \rightarrow\{\text { false }\} \\
& \text { alarm }:=\text { on } \\
& (\lceil V<100\rceil \wedge l>0.5) ; \nearrow(V<100)
\end{aligned}
$$

The proof outline indicates that to guarantee the simple invariant requirement, it is sufficient that algorithm $A$ does not assign a value bigger than 15 to $a$, or formally

$$
\{V<80\} A\{V<80 \wedge a \leq 15\}
$$

It is preferable to accelerate fast when the speed is low and accelerate slowly when the speed is high. Therefore, we calculate the value of $a$ based on function $0.1 * e^{100 /(v+20)}$. To this end, we let

$$
\{\text { true }\} A\left\{0.1 * e^{100 /(v+20)}-1 \leq a \leq 0.1 * e^{100 /(v+20)}\right\}
$$


Correctness of the system is maintained, because

$$
0.1 * e^{100 /(v+20)} \leq 15 \quad \text { for any } v \geq 0
$$

Finally, the control algorithm $A$ is developed. It can be verified using Hoare Logic which has been embedded.

$$
\begin{aligned}
& c:=100 /(v+20) \\
& k:=1 \\
& a:=0.1 \\
& r:=0.6 * 5 \\
&\left\{a=0.1 * \sum_{n=0}^{k-1} \frac{1}{n !}\left(\frac{100}{v+20}\right)^{n} \wedge r=0.6 * 5^{k} / k !\right\} \\
& \text { while } k \leq 5 \vee r \geq 1 \text { do } \\
& \quad a:=a+0.1 * c ; \\
& \quad k:=k+1 ; \\
& \quad c:=(100 * c) /((v+20) * k) ; \\
& r:=(5 * r) / k \\
& \text { od } \\
&\left\{a=0.1 * \sum_{n=0}^{k-1} \frac{1}{n !}\left(\frac{100}{v+20}\right)^{n} \wedge r=0.6 * 5^{k} / k ! \wedge k>6 \wedge r<1\right\} \\
&\left\{0.1 * e^{100 /(v+20)}-r \leq a \leq 0.1 * e^{100 /(v+20)}\right\} \\
&\left\{0.1 * e^{100 /(v+20)}-1 \leq a \leq 15\right\}
\end{aligned}
$$

\section{DISCUSSION}

This paper presents a theory for reasoning about Sequential Hybrid Programs. These include the usual sequential programming constructs as well as phase statements which are Extended Duration Calculus specifications of time dependent dynamics. A compositional semantics of SHP is given using an extension of the Duration Calculus. Some high level rules are defined for modular verification of properties like total correctness and invariance.

An initial formulation of these ideas was given in (Xu 1997). Since then, the base logic used for formalising the semantics has significantly evolved. The original formulation used infinite conjunctions and disjunctions, causing the logic to be more complex than necessary, and the treatment of infinite behaviours was somewhat cumbersome.

Based on several previous papers, the present logic $\mu$ SDCI incorporates super dense states, greatest and least fixed point operators, and infinite intervals. Duration Calculus with super dense states has been studied in (Zhou \&Hansen 1996), and in (Xu 1997). The fixed point operators have been introduced in Duration Calculus in (Pandya \& Ramakrishna 1995), and used to model the terminating and divergent behaviours of programs. Our approach 
to infinite intervals follows the work (Moszkowski 1995) in Interval Temporal Logic.

In the literature, hybrid systems are often represented as Automata or as Phase Transition Systems (Manna \& Pnueli 1993, Henzinger, Manna \& Pnueli 1994). In our approach, we work with a notation for hybrid systems which is closer to how hybrid systems are programmed. In particular, complex digital control algorithms can be directly incorporated in our framework as subprograms. Similar description languages have been proposed in ( $\mathrm{He} 1994$, Fix \& Schneider 1994, Zhou, Wang \& Ravn 1995), but they all assume that every action takes non-zero time. In contrast, we allow instantaneous actions, which together with recursion have led to complex behaviours such as termination, infinite executions, finite and instantaneous divergence. We formalise the semantics using a mixture of the greatest and the least fixed points. This appears to be novel as compared to the usual approach of using a single fixed point.

Using the logical semantics, we have derived several high level proof rules for establishing specific kinds of properties of hybrid systems. These rules provide a modular and syntax directed method for verification of SHP programs, extending to hybrid systems the design and verification paradigm, proposed originally by Dijkstra, Gries and Hoare for sequential programs. Well established methods such as Hoare Logic can be embedded within our proof method. These are especially useful and also complete for reasoning about complex discrete components incorporating algorithms for digital control. In contrast to most of the existing work which assumes that divergent behaviours do not exist, usually by ruling out instantaneous actions altogether or by an axiom which states that time is never bounded, we can prove that divergent behaviours indeed do not occur. Properties studied so far include total correctness and invariance. Further investigation is needed for other properties.

Our theory is developed for sequential systems. One way to reason about concurrent systems is to first transform them into sequential ones. The techniques in this paper can then be applied. However, this approach may lead to state explosion and therefore modular verification methods for concurrent hybrid systems should be studied. Adding concurrency, nevertheless, is nontrivial, as intermediate states arising during the control actions of one process can be significant for the other processes. This requires the logic to be able to describe the sequences of states occurring at a single time point, leading to the notion of the so-called weakly monotonic time, which was first studied in real-time temporal logic (Koymans 1992). A Duration Calculus of Weakly Monotonic Time has been proposed in (Pandya \& Dang 1996) where compositional semantics of concurrent systems have also been formalised, and in (Liu, Ravn \& Li 1998). In future, we plan to extend our theory to Concurrent Hybrid Programs using a logic similar to $\mu$ SDCI with weakly monotonic time.

Acknowledgements We thank a number of researchers for comments and 
discussions, especially R. Barua, M.R. Hansen, Dang Van Hung, B. von Karger, A. Ravn, W.-P. de Roever, Zhou Chaochen and anonymous referees.

\section{REFERENCES}

Apt, K. \& Olderog, E.-R. (1997), Verification of Sequential and Concurrent Programs (2nd edition), Springer-Verlag.

Back, R. (1980), Correctness Preserving Program Refinements: Proof Theory and Applications, Vol. 131 of Mathematical Center Tracts, Mathematical Centre, Amsterdam.

Fix, L. \& Schneider, F. (1994), Hybrid verification by exploiting environment, in H. Langmack, W.-P. de Roever \& J. Vytopil, eds, 'Formal Techniques in Real-Time and Fault-Tolerant Systems', LNCS 863, SpringerVerlag, pp. 1-18.

Hansen, M., Pandya, P. \& Zhou Chaochen. (1995), 'Finite divergence', Theoretical Computer Science 138, 113-139.

He Jifeng. (1994), From csp to hybrid systems, in A. Roscoe, ed., 'A Classical Mind, Eassy in Honour of C.A.R. Hoare', Prentice-Hall International, pp. 171-189.

Henzinger, T., Manna, Z. \& Pnueli, A. (1994), 'Temporal proof methodologies for timed transition systems', Information and Computation 112(2), 273-337.

Hoare, C. A. R. (1985), Communicating Sequential Processes, Prentice-Hall.

Koymans, R. (1992), Specifying Message Passing and Time-Critical Systems with Temporal Logic, LNCS 651, Springer-Verlag.

Kozen, D. \& Parikh, R. (1981), 'Results on the propositional $\mu$-calculus', Theoretical Computer Science 14, 113-118.

Liu, Z., Ravn, A. \& Li, X.-S. (1998), Verifying duration properties of timed transition systems, this volume.

Manna, Z. \& Pnueli, A. (1993), Verifying hybrid systems, in 'Hybrid Systems, R.L. Grossman, A. Nerode, A.P. Ravn, H. Rischel (Eds.)', LNCS 736, Springer-Verlag, pp. 36-59.

Morgan, C. (1990), Programming from Specifications, Prentice-Hall.

Moszkowski, B. (1985), 'A temporal logic for multilevel reasoning about hardware', IEEE Computer 18(2), 10-19.

Moszkowski, B. (1995), Compositional reasoning about projected and infinite time, in 'Proc. the First IEEE International Conference on Engineering of Complex Computer Systems (ICECCS'95)', IEEE Computer Society Press, pp. 238-245.

Pandya, P. \& Dang, V.H. (1996), Duration calculus with weakly monotonic time, Technical Report Detfors 6, UNU/IIST, P.O. Box 3058 Macau.

Pandya, P. \& Ramakrishna, Y. (1995), A recursive duration calculus, Technical report, CS-95/3, Computer Science Group, TIFR, Bombay.

Scott, D. \& Strachey, C. (1971), Toward a mathematical semantics for com- 
puter languages, in J. Fox, ed., 'Proc. Symp. Computer and Automata', Polytechnic Institute of Brooklyn Press, pp. 19-46.

Stirling, C. (1995), Modal and temporal logics, in S. Abramsky, D. M. Gabbay \& T. Maibaum, eds, 'Handbook of Logic in Computer Science Volume 2 Background: Computational Structures', Clarendon Press - Oxford, pp. 476-563.

Xu, Q.-W. (1997), Semantics and verification of extended phase transition systems in duration calculus, in O. Maler, ed., 'International Workshop on Hybrid and Real-Time Systems', LNCS 1201, Springer-Verlag, pp. 301-315.

Zhou Chaochen, \& Hansen, M. R. (1996), Chopping a point, in J.-F. He, J. Cooke \& P. Wallis, eds, 'Proc. BCS FACS 7th Refinement Workshop:Theory and Practice of System Design', Electronic Workshops in Computing, Springer-Verlag, Bath, U.K.

Zhou Chaochen, Hoare, C. \& Ravn, A. (1991), 'A calculus of durations', Information Processing Letters 40(5), 269-276.

Zhou Chaochen, Wang Ji, \& Ravn, A. (1995), A formal description of hybrid systems, in R. Alur, T. Henzinger \& E. Sontag, eds, 'Hybrid Systems III: Verification and Control', LNCS 1066, Springer-Verlag, pp. 511530.

Zhou Chaochen, Ravn, A. \& Hansen, M. (1993), An extended duration calculus for hybrid systems, in 'Hybrid Systems, R.L. Grossman, A. Nerode, A.P. Ravn, H. Rischel (Eds.)', LNCS 736, Springer-Verlag, pp. 36-59.

\section{BIOGRAPHY}

Pandya, Paritosh K. is a Reader in the Computer Science Department of the Tata Institute of Fundamental Research, Mumbai, India. He obtained a Ph.D. in Computer Science from Bombay University in 1988. He has worked as a research officer at Oxford University Computing Laboratory, U.K., and as visiting researcher at UNU/IIST, Macau. His research interests are logics of programs, analysis of real-time systems, concurrency theory and formal methods in programming.

Wang, Hanpin is a faculty member in the Department of Computer Science and Technology, Beijing University, P.R. China. He obtained a Ph.D. in Mathematical Logic from Beijing Normal University, P.R. China in 1993. He was a fellow of UNU/IIST from February 1997 to August 1997. His research interests are mathematical logic especially model theory, computational complexity and logics of programs.

$\mathrm{Xu}$, Qiwen is a Research Fellow of UNU/IIST. He obtained a D.Phil. in Computing from Oxford University Computing Laboratory, U.K., in 1992. He has worked as a research associate at Christian Albrechts Universität zu Kiel, Germany and Åbo Akademi, Finland. His research interests are program verification and refinement, theory of real-time and concurrent systems. 